\title{
Synthesis and In vitro Antifungal Evaluation of Benzoimidazolyl-piperazinyl-phenylmethanone Derivatives
}

\author{
RANI S. KANKATE ${ }^{1,2 *}$, PARAG S. GIDE ${ }^{2}$ and DEEPAK P. BELSARE ${ }^{3}$ \\ 'Department of Pharmaceutical Chemistry, Jawaharlal Nehru Technological University, \\ Kukatpally, Hyderabad, Telangana - 500085. \\ 2Department of Pharmaceutical Chemistry, Pune University, \\ M.E.T's Institute of Pharmacy, Nashik- 422003, India. \\ ${ }^{3}$ Department of Pharmaceutical Chemistry, Pune University, NDMVPS college of Pharmacy, \\ Nashik-422001, Maharashtra, India. \\ *Corresponding author E-mail: ranipharmacy@gmail.com, paraagide@gmail.com
}

http://dx.doi.org/10.13005/ojc/300446

(Received: October 15, 2014; Accepted: December 09, 2014)

\begin{abstract}
Benzimidazole and piperazines are the important pharmacophores in the structures of many antifungal compounds. Further, the phenylmethanone are also a unique class of compounds whose antifungal profile is not much exploited. So to exploit their antifungal potential we have selected these three combinations and framed the novel parent structure for our research work. In this study a novel series of benzimidazoles derivatives was synthesized by microwave irradiation and characterized by ${ }^{1} \mathrm{H}$ NMR, ${ }^{13} \mathrm{C}$ NMR, Infra Red (IR), and Mass Spectroscopy (MS), and by elemental analysis. The screening of compound for in vitro (turbidimetric method) antifungal activity against C.albicans revealed activity in many of the compounds as comparable to that of ketoconazole.
\end{abstract}

Key words: Antifungal activity, Benzimidazole, Piperazine and Phenylmethanone.

\section{INTRODUCTION}

During the past two decades, the frequency of systemic infections has increased dramatically along with the number of invasive, mostly opportunistic, fungal species carrying infectious diseases ${ }^{1}$. In clinic, there are very few antifungal agents that can be used for life threatening fungal infections. Clinically available antifungal agent include the following: polyenes (such as amphotericin B and nyastatin) ${ }^{2}$ azoles (suchas ketoconazole, fluconazole, itraconazole and voriconazole) ${ }^{3}$ echinocandins(such as caspofungin and micafungin) ${ }^{4}$. None of the existing systemic antifungal satisfies medical need completely; there are weaknesses in spectrum, potency, safety and pharmacokinetic properties. Moreover, severe resistance has been observed 
for triazoles ${ }^{5}$. Along with this the cost of treatment for such infections is also on a high. So an ideal, which has a broad spectrum of activity and has a low on side effects, is to be found having a cheaper cost of treatment.

Therefore, there is an emergent need to develop new antifungal drugs with novel chemical structures and novel modes of action. The field of antifungal development is wide open for extensive research work.

Benzimidazole derivatives possess great importance in medicinal chemistry due to wide variety of pharmacogical activity ${ }^{6,7}$ in controlling cardiovascular diseases ${ }^{8}$, anticancer properties ${ }^{9}$, antiinflamatory ${ }^{10}$, antibacterial ${ }^{11}$, antifungal ${ }^{12}$, antidiabetic ${ }^{13}$ and anti HIV ${ }^{14}$ activity and some benzimidazoles are antioxidant ${ }^{15-16}$. The special benzene-fused imidazole ring could readily interact with various active targets in biological system via diverse noncovalent interactions like hydrogen bonds, coordination, ion dipole, cation À-À stacking and hydrophobic effect as well as van der Waals force, therefore benzimidazole-based derivatives exhibit various bioactivities ${ }^{17}$. Benzimidazole and its derivatives are used in organic synthesis and vermicides or fungicides as they inhibit the action of certain microorganisms. Examples of benzimidazole class fungicides include benomyl, carbendazim, chlorfenazole, cypendazole, debacarb, fuberidazole, furophanate, mecarbinzid, rabenzazole, thiabendazole, thiophanate. A perusal of literature revealed that benzimidazol, piperazine ring and phenylmethanone were significant structural moieties for antifungal activity ${ }^{18-21}$.

Considering the above scenario of biological profile we have designed, synthesized and evaluated for antifungal activity series of benzoimidazolyl-piperazinyl-phenylmethanone derivatives.

\section{RESULTS AND DISCUSSION}

The syntheses of compounds 11a-11x are outlined in Scheme 1.

The N1-methyl/ethyl-2-nitroaniline $\mathbf{2} \mathbf{a}-\mathbf{b}$ was synthesized by using 2-fluronitrobenzene $\mathbf{1}$, methylamine/ethylamine and DMF as solvent in microwave [22]. The nitro group of phenyl ring $\mathbf{2 a - b}$ was further reduced to amino group in presence zinc and ammonium chloride to give $\mathbf{3} \mathbf{a}-\mathbf{b}$ [23].Compounds $\mathbf{3 a - b / 4 a - b}$ and lactic/mandelic acid 5a-b dissolved in methanol and reflux in microwave to give $6 a-f$.Then activation of carboxylic functional group of 6 a-f was carried out by using thionyl

Table 1: Effect of synthesized compounds against $C$.albicans by serial dilution method

\begin{tabular}{|c|c|c|c|c|c|c|c|c|}
\hline \multirow{2}{*}{$\begin{array}{l}\text { S. } \\
\text { No. }\end{array}$} & \multirow[t]{2}{*}{ Compounds } & \multicolumn{7}{|c|}{ Concentration of compound required for inhibition $(\mu \mathrm{mol} / \mathrm{ml})$} \\
\hline & & 0.5 & 0.25 & 0.125 & 0.0625 & 0.0312 & 0.015 & 0.007 \\
\hline 1. & $11 a$ & - & - & + & + & + & + & + \\
\hline 2. & $11 b$ & - & - & - & + & + & + & + \\
\hline 3. & $11 \mathrm{c}$ & - & - & - & + & + & + & + \\
\hline 4. & $11 d$ & - & - & - & + & + & + & + \\
\hline 5. & $11 \mathrm{e}$ & - & - & - & - & - & + & + \\
\hline 6. & $11 f$ & - & - & - & - & + & + & + \\
\hline 7. & $11 \mathrm{~g}$ & - & - & - & - & + & + & + \\
\hline 8. & $11 \mathrm{~h}$ & - & - & - & + & + & + & + \\
\hline 9. & $11 i$ & - & - & - & + & + & + & + \\
\hline 10. & $11 \mathrm{j}$ & - & - & - & - & + & + & + \\
\hline 11. & $11 \mathrm{k}$ & - & - & - & - & - & + & + \\
\hline 12. & 111 & - & - & - & - & - & - & + \\
\hline 13. & Ketoconazole & - & - & - & & - & - & - \\
\hline
\end{tabular}

(-)Indicates absence of growth; (+) Indicates presence of growth; K: Ketoconazole 
chloride to give 7a-f.Piperazine 8 and benzoyl chloride 9a-c were dissolved in $N, N$ dimethylformamide and reaction mixture stirred for $6 \mathrm{~h}$ at $80^{\circ} \mathrm{C}$ on a magnetic stirrer to give $10 \mathrm{a}-$ c.Further 10a-c condensed with $7 \mathrm{a}-\mathrm{f}$ to get target compounds 11a-X.

\section{Reagents and conditions}

(a) $\mathrm{K}_{2} \mathrm{CO}_{3}, \mathrm{DMF}$, alkylamine; (b) ammonium chloride, zinc dust; (c) mono chloro acetic acid, methanol, reflux; (d) lactic acid/mandelic acid, methanol, reflux; (e) thionyl chloride; (f) and (g) N,N-Dimethylformamide, $\mathrm{K}_{2} \mathrm{CO}_{3}$.

\section{Invitro Antifungal Activity}

Antifungal activity of the synthesized compounds was tested against different fungal spores in vitro. In vitro antifungal activity was evaluated using the tube dilution method (turbidimetric method). The turbidimetric method [24] depends on the inhibition of growth in a microbial culture of uniform solution containing the drug in fluid medium favorable for rapid growth. In this method, minimal inhibitory concentration (MIC) of the antifungal agent was determined. The MIC is the lowest concentration of an antimicrobial agent that inhibits the test organism. The growth in the tube was observed visually for turbidity and inhibition was determined by the absence of growth. In the present study, C.albicans (ATCC10235) was used to investigate the activity. Ketoconazole and Dimethyl sulphoxide were used as standard and solvent respectively.

The antifungal activity clearly indicated that 5-chloro substituted benzimidazole derivatives 11 I has a good antifungal activity as compared with the other twelve compounds at $0.015 \mu$ mole $/ \mathrm{ml}$ which is closer to ketoconazole activity as far as the in vitro results are concerned. Compounds $11 \mathrm{e}$ and $11 \mathrm{k}$ demonstrated equivalent activity against C. albicans and had an MIC of $0.03 \mu \mathrm{mole} / \mathrm{ml}$. The other compounds showed decreased in antifungal activity those are lack of asymmetric center at $\mathrm{C} 2$ and unsubstituted $\mathrm{N}-1$ position of benzimidazole.<smiles>Nc1ccc(-c2ccc(N(N)c3ccc([N+](=O)[O-])cc3)cc2)cc1N</smiles>

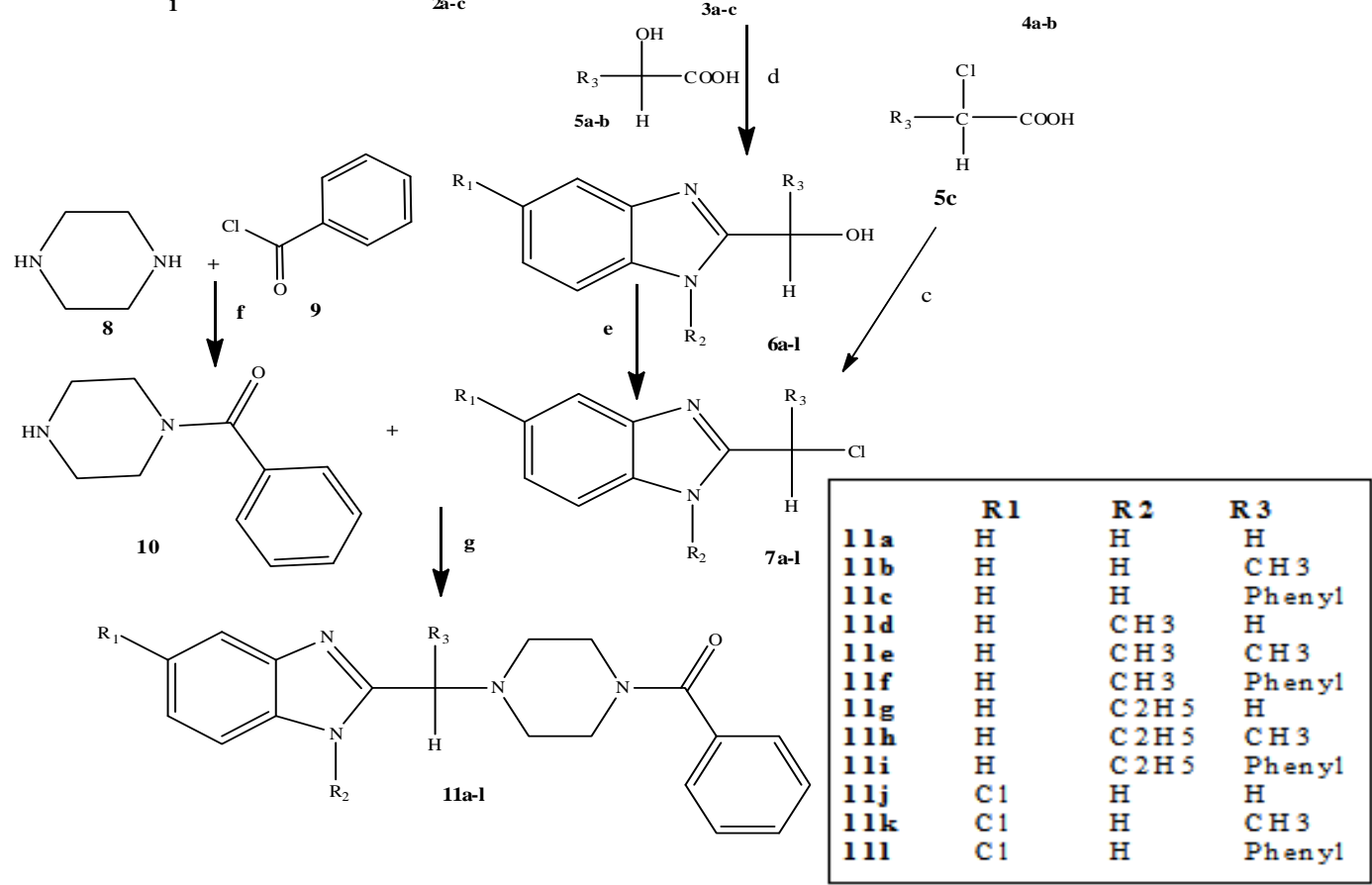

Scheme 1: Synthesis of tertiary amine type of substituted benzimidazole derivatives 


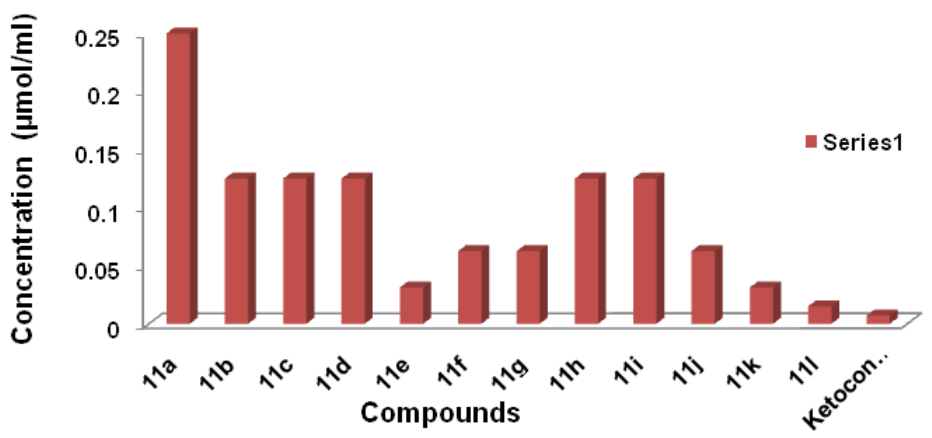

Fig. 1: In vitro Antifungal activity against Candida albicans

\section{EXPERIMENTAL}

All materials were procured from Sigma Aldrich and Merck specialties Pvt. Ltd. (Mumbai. India). Solvents were dried and distilled being used. Anhydrous sodium sulphate was used to dry the solvents. Thin Layer Chromatography (TLC) analyses were carried out on aluminum plates (Merck) precoated with silica gel 60 F254 (0.2 mm), and spots were visualized with $U V$ light and $I_{2}$. Liquid intermediates were checked for purity using Gas chromatography (Pack column SE-30, OV-101, and capillary column BP-5). Gravity column chromatography was performed using silica gel (Merck 60). Melting points were taken in open glass capillary using Elico melting point apparatus and were uncorrected. Infra red (IR) spectra were recorded on $\mathrm{KBr}$ pellets on a Shimadzu 1000 FTIR spectrometer in the range of $4000-200 \mathrm{~cm}^{-1}$, Resolution 2.0 with number of scan - 45 . Apodization; Happ-Genzel. Proton $\left({ }^{1} \mathrm{H}\right)$ Nuclear Magnetic Resonance (NMR) and ${ }^{13} \mathrm{C}$ NMR spectra of compounds were recorded on Bruker Advance II 400 NMR Spectrophotometer using $\mathrm{CDCl}_{3}$ solvent, at SAIF, Punjab University, Chandigarh. Mass spectra of compounds were recorded on API 4000 $Q$ TRAP LC/MS/MS system using electron spray ionization positive ion mass spectrometric technique, at NHRDF, Chitegaon, Nashik. Elemental analyses were performed on a Perkin-Elmer 2400 Analyser and are within $\pm 0.4 \%$ of theoretical values.

The method followed for the synthesis of benzimidazole derivatives has been elaborated here

N1-methyl/ethyl-2-nitroaniline (2) was synthesized by using $25 \mathrm{ml}$ (33.5 gm, $0.24 \mathrm{~mol}$ ) of 2- fluronitrobenzene (1) dissolved in DMF and anhydrous $\mathrm{K}_{2} \mathrm{CO}_{3}$ was added to it. $115 \mathrm{ml}(6 \mathrm{~mol})$ of methylamine/ethylamine was slowly added it through dropping funnel in cold condition. After complete addition of methylamine/ethylamine solution the reaction mixture was kept in microwave for $10 \mathrm{~min}$. The completion of reaction was checked by monitoring TLC. After completion, the reaction mixture was poured into ice cold water with stirring and extracted with the product with ether, the ether layer was separated and dried with sodium sulphate, and then ether was removed by distillation to get products $2 \mathrm{a}, 2 \mathrm{~b}$.

ynthesis of N1- methyl/ethyl-o-Phenylenediamine (3a, 3b)

$\mathrm{N}^{1}$ - methyl/ethyl-o-Phenylenediamine (5 mM), Zinc dust (0.25 gm) and ammonium chloride $(10 \mathrm{mM})$ in $5 \mathrm{ml}$ water were mixed thoroughly in a small beaker $(25 \mathrm{ml})$. The reaction solid mixture was placed in a microwave oven (300Watt) at 50\% power level for 8 to $15 \mathrm{~min}$; the progress of the reaction was monitored by checking the solubility of the solid reaction mixture product in dilute $\mathrm{HCl}$, when the entire organic solid dissolved it was filtered to separate Zinc dust, and neutralized with aqueous solution. The solid was separated by filtration and recrystallized from an appropriate solvent (aqueous Ethanol).

\section{Synthesis of 5-H/Chloro-1-alkyl-2-(a-hydroxyethyl} /benzyl)benzo[d] imidazoles (6a-I)

In a typical experiment, $(0.163 \mathrm{~m}) 3 \mathrm{a}-\mathrm{b}$ or $4 a-b,(0.170 \mathrm{~m})$ of lactic/mandelic $5 a-b$ / monochloroacetic acid $5 \mathrm{c}$ and $25 \mathrm{ml}$ of methanol was taken in a RBF and then placed in microwave irradiation at $350 \mathrm{~W}$ for $15 \mathrm{~min}$. The reaction was 
monitored by TLC. A test portion was added in water and basified with ammonia solution. The solid was extracted with ether and TLC of this ether extract was done to check for completion of reaction. After completion, the reaction mixture was poured in icecold water. It was then basified with concentrated ammonia solution. The solid precipitate was filtered immediately and dried.

Synthesis of 5-H/Chloro-1-alkyl-2-(a-chloroethyl/ benzyl)benzo[d] imidazoles (6a-l)

The activation of carboxylic function was carried out by using an excess of thionyl chloride and catalytic amount of dimethyl formamide placed in an ice cold water bath. To this mixture, $15 \mathrm{gm} \mathrm{2-}$ a-hydroxy benzyl/ethyl-N ${ }^{1}$ - alkyl-benzimidazole was added slowly with occasional stirring. This mixture was then placed in microwave irradiation at $250 \mathrm{~W}$ for $10 \mathrm{~min}$. Excess thionyl chloride was recovered under vacuum on a water bath. To the residue dry dioxane was added and stirred for $30 \mathrm{~min}$. Dioxane was recovered under vacuum to get the final product.

Synthesis of phenyl(piperazine-1-yl)methanone from anhydrous Piperazine and Benzoyl chloride 10.

A solution of Benzoyl chloride 9 (8.26 g, $0.058 \mathrm{~mol})$ and Piperazine 8 (10.12 g, 0.117mol) in $\mathrm{N}, \mathrm{N}$ dimethylformamide was taken in a RBF $\mathrm{K}_{2} \mathrm{CO}_{3}$ $(3 \mathrm{gm})$ was added to the reaction mixture. The reaction mixture was stirred for $8 \mathrm{hr}$ at $80^{\circ} \mathrm{C}$ on a magnetic stirrer (heat + stirring). The progress of the reaction was monitored by thin layer chromatography (TLC). Upon completion of the reaction, water was added to the reaction mixture and the product extracted by shaking the reaction mixture with dichloromethane in a separating funnel.The dichloromethane layer was washed successively with water and brine, dried over anhydrous sodium sulfate. Evaporation of the solvent gave the product $\mathbf{1 0}$. Recrystallized with chloroform.

Synthesis of Substituted Benzimidazole Derivatives Bearing 1-[(4-phenyl)carbonyl] piperazinyl alkyl benzo(d) imidazole derivatives 11a-I

A solution of 2-chloroalkyl/aryl substituted with or without $\mathrm{N}$-substitution as well as with or without 5 and/or 6-substituted benzimidazole derivative $(1.75 \mathrm{~g}, 0.01051 \mathrm{~mol})$ and 1-[(4-phenyl) carbonyl]piperazine $(3 \mathrm{~g}, 0.0105 \mathrm{~mol})$ in $\mathrm{N}, \mathrm{N}$ dimethylformamide was taken in a RBF. $\mathrm{K}_{2} \mathrm{CO}_{3}(2 \mathrm{gm}$, ) was added to the reaction mixture. The reaction mixture was stirred for $8 \mathrm{~h}$ at $80^{\circ} \mathrm{C}$ on a magnetic stirrer (heat + stirring). The progress of the reaction was monitored by thin layer chromatography (TLC). Upon completion of the reaction, water was added to the reaction mixture and the product extracted by shaking the reaction mixture with dichloromethane in a separating funnel.The dichloromethane layer was washed successively with water and brine, dried over anhydrous sodium sulfate. Evaporation of the solvent gave the product. 11a-I Recrystallized with various solvent like chloroform, ethanol, methanol.

(4-((1H-benzo[d]imidazol-2-yl) methyl) piperazin1-yl)(Phenyl) methanone (11a)

Compound 11a was obtained as a white solid (yield: 74.35\%; MP:224-226; IR (KBr): 3220 $(\mathrm{N}-\mathrm{H}), 2365(\mathrm{C}-\mathrm{H}), 1670(\mathrm{C}=\mathrm{O}), 1604(\mathrm{C}=\mathrm{N})$, 1556(C=C), 1232 (C-N) cm-1; ${ }^{1} \mathrm{H}$ NMR (300 MHz, CDCl3) $\delta$ ppm: 3.45-3.51(m, 4H,PIP-H), 4.34(s, 2H, $\left.-\mathrm{CH}_{2}-\right), 4.79(\mathrm{~s}, 1 \mathrm{H}, \mathrm{N}-\mathrm{H}), 7.30-7.45(\mathrm{~m}, 4 \mathrm{H}, \mathrm{Ar}-\mathrm{H}$ of Benzim) 7.89-8.07 (4H, Ar-H of benzoyl) ; ${ }^{13} \mathrm{C}-\mathrm{NMR}$ (400 MHz, CDCl3, TMS): $\delta 52.14(\mathrm{CH} 2), 55.4(\mathrm{CH} 2)$, $61.7\left(\mathrm{CH}_{2}\right), 117$ (arom. $\left.\mathrm{CH}\right), 124$ (arom. $\left.\mathrm{CH}\right), 129$ (arom. $\mathrm{CH}$ ), 133.5 (quat. C), 139.8 (quat. C), 145.6 (quat. C), 171.6 (carbonyl C).ESI-MS m/ z:320,243,189,131.Anal. Calcd. for C19H20N4O (320): C, 71.23; H, 6.29; N, 17.49; O, 4.99. Found: C, 71.10; H, 6.23; N, 17.43; O, 4.91.

\section{(4-(1-(1H-benzo[d]imidazol-2-yl) ethyl) piperazin-} 1-yl)(Phenyl) methanone (11b)

Compound $11 \mathrm{~b}$ was obtained as a white solid (yield: 71.25\%; MP:234-236; IR (KBr): 3227 $(\mathrm{N}-\mathrm{H}), 2361(\mathrm{C}-\mathrm{H}), \quad 1674(\mathrm{C}=\mathrm{O}), 1601(\mathrm{C}=\mathrm{N})$, 1550(C=C), 1239 (C-N) cm-1; ${ }^{1} \mathrm{H}$ NMR (300 MHz, $\left.\mathrm{CDCl}_{3}\right) \delta$ ppm:1.24(d,1H,-CH-), 3.25-3.31(m, 4H,PIP-H), 4.13(q, 3H,- $\mathrm{CH}_{3}$ ), 5.09(s, $\left.1 \mathrm{H}, \mathrm{N}-\mathrm{H}\right), 7.28-$ $7.35(\mathrm{~m}, 4 \mathrm{H}, \mathrm{Ar}-\mathrm{H}$ of Benzim) 7.79-8.01 (4H, Ar - $\mathrm{H}$ of benzoyl) ; ${ }^{13} \mathrm{C}-\mathrm{NMR}$ (400 MHz, $\left.\mathrm{CDCl}_{3}, \mathrm{TMS}\right): \delta$ $14.8\left(\mathrm{CH}_{3}\right), 51.11\left(\mathrm{CH}_{2}\right), 53.2\left(\mathrm{CH}_{2}\right), 61.5\left(\mathrm{CH}_{2}\right)$, 115(arom. CH), 123 (arom. CH), 128 (arom. CH), 131.6 (quat. C), 135.5 (quat. C), 144.2 (quat. C), 172.5 (carbonyl C); ESI-MS m/z: 334,319, 257,145,117. Anal. Calcd. for $\mathrm{C}_{20} \mathrm{H}_{22} \mathrm{~N}_{4} \mathrm{O}$ (334): $\mathrm{C}$, 71.83; H, 6.63; N, 16.75; O, 4.78. Found: C, 71.80; H, $6.61 ; \mathrm{N}, 16.79 ; \mathrm{O}, 4.73$. 
(4-((1H-benzo[d]imidazol-2-yl)(phenyl) methyl)piperazin-1-yl)(Phenyl) methanone (11c) Compound $11 \mathrm{C}$ was obtained as a white solid (yield: 65.45\%; MP:284-286; IR (KBr): 3219 $(\mathrm{N}-\mathrm{H}), 2359(\mathrm{C}-\mathrm{H}), \quad 1671(\mathrm{C}=\mathrm{O}), 1605(\mathrm{C}=\mathrm{N})$, 1548(C=C), 1236 (C-N) cm-1; ${ }^{1} \mathrm{H}$ NMR $(300 \mathrm{MHz}$, $\left.\mathrm{CDCl}_{3}\right) \delta \mathrm{ppm}: 3.10-3.14(\mathrm{~m}, 4 \mathrm{H}, \mathrm{PIP}-\mathrm{H}), 4.89(\mathrm{~s}, 1 \mathrm{H}, \mathrm{N}-$ $\mathrm{H}$ ), 5.04(s, 1H, C-H), 7.28-8.05 (Complex m, 4H, Ar $-\mathrm{H}) ;{ }^{13} \mathrm{C}-\mathrm{NMR}\left(400 \mathrm{MHz}, \mathrm{CDCl}_{3}, \mathrm{TMS}\right): \delta 50.11\left(\mathrm{CH}_{2}\right)$, 52.4 $\left(\mathrm{CH}_{2}\right), 71.5(\mathrm{CH}), 118$ (arom. $\mathrm{CH}$ ), 125 (arom. $\mathrm{CH}$ ), 130 (arom. $\mathrm{CH}$ ), 131.2 (arom. $\mathrm{CH}$ ), 132.8 (quat. C), 136.5 (quat. C), 141.9 (quat. C), 144.3 (quat. C), 169.5; (carbonyl C); ESI-MS m/z:396,319, 291,279,207.Anal. Calcd. for $\mathrm{C}_{25} \mathrm{H}_{24} \mathrm{~N}_{4} \mathrm{O}$ (396): C, 75.69; H, 6.14; N, 14.11; O, 4.08 Found: C, 75.73; H, $6.10 ; \mathrm{N}, 14.13 ; \mathrm{O}, 4.04$.

(4-((1-methyl-1H-benzo[d]imidazol-2-yl) methyl) piperazin-1-yl)(Phenyl) methanone (11d)

Compound $11 \mathrm{~d}$ was obtained as a white solid (yield: 68\%; MP:212-215; IR (KBr): $3225(\mathrm{~N}-$ $\mathrm{H}), \quad 2354(\mathrm{C}-\mathrm{H}), \quad 1668(\mathrm{C}=\mathrm{O}), 1608(\mathrm{C}=\mathrm{N})$, 1546(C=C), $1230(\mathrm{C}-\mathrm{N}) \mathrm{cm}^{-1}$; ${ }^{1} \mathrm{H}$ NMR $(300 \mathrm{MHz}$, $\left.\mathrm{CDCl}_{3}\right) \delta \mathrm{ppm}: 3.01-3.05(\mathrm{~m}, 4 \mathrm{H}, \mathrm{PIP}-\mathrm{H}), 3.19(\mathrm{~s}, 3 \mathrm{H}, \mathrm{N}-$ $\left.\mathrm{CH}_{3}\right), 5.07(\mathrm{~s}, 1 \mathrm{H}, \mathrm{C}-\mathrm{H}), 7.28-7.35(\mathrm{~m}, 4 \mathrm{H}, \mathrm{Ar}-\mathrm{H})$, 7.88-7.95 ( m, 4H, Ar - H); ${ }^{13} \mathrm{C}-\mathrm{NMR}(400 \mathrm{MHz}$, $\mathrm{CDCl} 3, \mathrm{TMS}): \delta 30.8\left(\mathrm{CH}_{3}\right), 51.11\left(\mathrm{CH}_{2}\right), 55.2\left(\mathrm{CH}_{2}\right)$, $60.5\left(\mathrm{CH}_{2}\right), 119$ (arom. $\mathrm{CH}$ ) , 122.5 (arom. $\mathrm{CH}$ ), 128.7 (arom. CH), 132.6 (quat. C), 135.4 (quat. C), 144.5 (quat. C), 173.5 (carbonyl C); ESI-MS m/z: $334,319,229,203,145$. Anal. Calcd. For $\mathrm{C}_{20} \mathrm{H}_{22} \mathrm{~N}_{4} \mathrm{O}$ (334): C, 71.83; H, 6.63; N, 16.75; O, 4.72 Found: C, $71.79 ; \mathrm{H}, 6.61 ; \mathrm{N}, 16.70 ; \mathrm{O}, 4.74$.

(4-(1-(1-methyl-1H-benzo[d]imidazol-2-yl) ethyl) piperazin-1-yl)(Phenyl) methanone (11e)

Compound $11 \mathrm{e}$ was obtained as a white solid (yield: 70.15\%; MP:222-224; IR (KBr): 3205 $(\mathrm{N}-\mathrm{H}), 2355(\mathrm{C}-\mathrm{H})$, 1663( $\mathrm{C}=\mathrm{O}), 1610(\mathrm{C}=\mathrm{N})$, 1541(C=C), 1235 (C-N) cm-1; ${ }^{1} \mathrm{H}$ NMR $(300 \mathrm{MHz}$, $\mathrm{CDCl} 3) \delta$ ppm: $1.20(\mathrm{~d}, 1 \mathrm{H},-\mathrm{CH}-), 3.22-3.29(\mathrm{~m}$, $4 \mathrm{H}, \mathrm{PIP}-\mathrm{H}), 3.83(\mathrm{~s}, 3 \mathrm{H}, \mathrm{N}-\mathrm{CH} 3), 4.16(\mathrm{q}, 3 \mathrm{H},-\mathrm{CH} 3$ ), 7.28-7.35 (m, 4H, Ar - $\mathrm{H}$ of Benzim) 7.79-8.01 (4H, $\mathrm{Ar}-\mathrm{H}$ of benzoyl); ${ }^{13} \mathrm{C}-\mathrm{NMR}\left(400 \mathrm{MHz}, \mathrm{CDCl}_{3}, \mathrm{TMS}\right)$ :

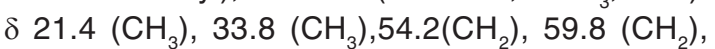
$62.10(\mathrm{CH}), 115$ (arom. CH), 121.9 (arom. $\mathrm{CH}), 127.9$ (arom. $\mathrm{CH}$ ), 134.5(quat. C), 136.6 (quat. C), 147.6 (quat. C), 171.2 (carbonyl C); ESI-MS m/z: 348,333, 319,217. Anal. Calcd. For $\mathrm{C}_{21} \mathrm{H}_{24} \mathrm{~N}_{4} \mathrm{O}$ (348): C, 72.39; $\mathrm{H}, 6.94$; N, 16.08; O, 4.59 Found: C, 72.34; H, 6.97;
N, $16.05 ;$ O, 4.62.

(4-((1-methyl-1H-benzo[d]imidazol-2yl)(phenyl)methyl)piperazin-1-yl)(Phenyl) methanone (11f)

Compound 11f was obtained as a white solid (yield: 66.55\%; MP:291-293; IR (KBr): 3219 $(\mathrm{N}-\mathrm{H}), 2359(\mathrm{C}-\mathrm{H}), \quad 1671(\mathrm{C}=\mathrm{O}), 1605(\mathrm{C}=\mathrm{N})$, 1548(C=C), 1236 (C-N) cm ${ }^{-1}$; ${ }^{1} \mathrm{H}$ NMR $(300 \mathrm{MHz}$, $\left.\mathrm{CDCl}_{3}\right) \delta$ ppm:3.14-3.19(m, 4H,PIP-H), 3.83(s, 3H,N$\mathrm{CH}_{3}$ ), 5.01(s, $1 \mathrm{H}, \mathrm{C}-\mathrm{H}$ ), 7.39-8.13 ( Complex m, 4H, $\mathrm{Ar}-\mathrm{H}$ ); ${ }^{13} \mathrm{C}$-NMR (400 MHz, $\mathrm{CDCl}_{3}$, TMS): $\delta 35.4$ $\left(\mathrm{CH}_{3}\right), 51.15\left(\mathrm{CH}_{2}\right), 54.7\left(\mathrm{CH}_{2}\right), 74.5(\mathrm{CH}), 116$ (arom. $\mathrm{CH}$ ), 126 (arom. $\mathrm{CH}$ ), 132.3 (arom. CH), 135.6 (arom. $\mathrm{CH}$ ), 133.1 (quat. C), 137.4 (quat. C), 142.8 (quat. C), 143.2 (quat. C), 168.3; (carbonyl C); ESI-MS m/z: 410,395,333,305,207.Anal. Calcd. For $\mathrm{C}_{26} \mathrm{H}_{26} \mathrm{~N}_{4} \mathrm{O}$ (410): C, 76.07; H, 6.38; N, 13.65; O, 3.90 Found: C, 76.04; H, 6.35; N, 13.57; O, 3.87 .

(4-((1-ethyl-1H-benzo[d]imidazol-2-yl) methyl)piperazin-1-yl)(Phenyl) methanone (11g)

Compound $11 \mathrm{~g}$ was obtained as a white solid (yield: 74.35\%; MP:224-226; IR (KBr): 3220 $(\mathrm{N}-\mathrm{H}), 2365(\mathrm{C}-\mathrm{H}), 1670(\mathrm{C}=\mathrm{O}), 1604(\mathrm{C}=\mathrm{N})$, 1556 $(\mathrm{C}=\mathrm{C}), 1232(\mathrm{C}-\mathrm{N}){ }^{\mathrm{cm}-1} ;{ }^{1} \mathrm{H}$ NMR $(300 \mathrm{MHz}$, $\left.\mathrm{CDCl}_{3}\right) \delta$ ppm:1.23(t,2 $\left.\mathrm{H}, \mathrm{N}-\mathrm{CH}_{2}-\right), 3.35-3.41(\mathrm{~m}$, $4 \mathrm{H}, \mathrm{PIP}-\mathrm{H}$ ), $4.10\left(\mathrm{q}, 3 \mathrm{H},-\mathrm{CH}_{3}\right.$ ), 4.31(s, $\left.2 \mathrm{H},-\mathrm{CH} 2-\right)$, 7.35-7.42 (m, 4H, Ar - $\mathrm{H}$ of Benzim) 7.84-8.05 $(4 \mathrm{H}$, $\mathrm{Ar}-\mathrm{H}$ of benzoyl) $;{ }^{13} \mathrm{C}-\mathrm{NMR}(400 \mathrm{MHz}, \mathrm{CDCl} 3, \mathrm{TMS})$ : $\delta 14.6\left(\mathrm{CH}_{3}\right), 38.6\left(\mathrm{CH}_{2}\right), 49.3\left(\mathrm{CH}_{2}\right), 55.4\left(\mathrm{CH}_{2}\right), 56.7$ $\left(\mathrm{CH}_{2}\right), 118$ (arom. $\mathrm{CH}$ ), 122.8 (arom. $\mathrm{CH}$ ), 130.8 (arom. $\mathrm{CH}$ ), 132.7 (quat. C), 138.6 (quat. C), 149.5 (quat. C), 174.3 (carbonyl C); ESI-MS m/z: $348,319,243,159$.Anal. Calcd. For $\mathrm{C}_{21} \mathrm{H}_{24} \mathrm{~N}_{4} \mathrm{O}$ (348): C, 72.39; H, 6.94; N, 16.08; O, 4.59 Found: C, 72.15; $\mathrm{H}, 6.81 ; \mathrm{N}, 16.05 ; \mathrm{O}, 4.53$.

(4-(1-(1-ethyl-1H-benzo[d]imidazol-2-yl) ethyl) piperazin-1-yl)(Phenyl) methanone (11h)

Compound 11b was obtained as a white solid (yield: 64\%; MP:275-277; IR (KBr): $3227(\mathrm{~N}$ $\mathrm{H}), 2361(\mathrm{C}-\mathrm{H}), 1674(\mathrm{C}=\mathrm{O}), 1601(\mathrm{C}=\mathrm{N}), 1550$ $(\mathrm{C}=\mathrm{C}), 1239(\mathrm{C}-\mathrm{N}) \mathrm{cm}^{-1} ;{ }^{1} \mathrm{H}$ NMR $\left(300 \mathrm{MHz}, \mathrm{CDCl}_{3}\right)$ $\delta$ ppm:1.22-127( $\left.\mathrm{m}, 3 \mathrm{H},-\mathrm{CH}_{3}\right), 3.23-3.28(\mathrm{~m}, 4 \mathrm{H}, \mathrm{PIP}-$ $\mathrm{H}), 4.10\left(\mathrm{q}, 3 \mathrm{H},-\mathrm{CH}_{3}\right), 5.02(\mathrm{~s}, 1 \mathrm{H}, \mathrm{N}-\mathrm{H}), 7.26-7.32$ $(\mathrm{m}, 4 \mathrm{H}, \mathrm{Ar}-\mathrm{H}$ of Benzim) 7.95-8.01 $(4 \mathrm{H}, \mathrm{Ar}-\mathrm{H}$ of benzoyl) ; ${ }^{13} \mathrm{C}-\mathrm{NMR}(400 \mathrm{MHz}, \mathrm{CDCl} 3, \mathrm{TMS}): \delta 14.6$ $\left(\mathrm{CH}_{3}\right), 20.8\left(\mathrm{CH}_{3}\right), 48.7\left(\mathrm{CH}_{2}\right), 51.2\left(\mathrm{CH}_{2}\right), 53.1\left(\mathrm{CH}_{2}\right)$, $60.2(\mathrm{CH}), 120$ (arom. CH), 125.8 (arom. $\mathrm{CH}$ ), 
132(arom. CH), 133(quat. C), 134.5(quat. C), 150.6 (quat. C), 171.2 (carbonyl C); ESI-MS m/z: $362,347,333,319,257,145$. Anal. Calcd. For $\mathrm{C}_{22} \mathrm{H}_{26} \mathrm{~N}_{4} \mathrm{O}(362): \mathrm{C}, 72.90 ; \mathrm{H}, 7.23 ; \mathrm{N}, 15.46 ; \mathrm{O}, 4.41$ Found: C, 72.92; H, 7.20; N, 15.49; O, 4.39.

(4-((1-ethyl-1H-benzo[d]imidazol-2-yl) (phenyl) methyl) piperazin-1-yl)(Phenyl) methanone (11i)

Compound $11 \mathrm{i}$ was obtained as a white solid (yield: 67\%; MP:258-260; IR (KBr): 3219 (N$\mathrm{H}), \quad 2359(\mathrm{C}-\mathrm{H}), \quad 1671(\mathrm{C}=\mathrm{O}), 1605(\mathrm{C}=\mathrm{N})$, 1548(C=C), $1236(\mathrm{C}-\mathrm{N}) \mathrm{cm}-1 ;{ }^{1} \mathrm{H}$ NMR $(300 \mathrm{MHz}$, $\left.\mathrm{CDCl}_{3}\right) \delta \mathrm{ppm}: 1.24\left(\mathrm{t}, 2 \mathrm{H}, \mathrm{N}-\mathrm{CH}_{2}-\right), 3.12-3.16(\mathrm{~m}$, $4 \mathrm{H}, \mathrm{PIP}-\mathrm{H}), 3.81$ (q, 3H,C-CH $\left.{ }_{3}\right), 4.98(\mathrm{~s}, 1 \mathrm{H}, \mathrm{C}-\mathrm{H})$, 7.49-8.11 ( Complex m, 4H, Ar-H); 13C-NMR (400

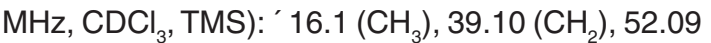
$\left(\mathrm{CH}_{2}\right), 53.2\left(\mathrm{CH}_{2}\right), 68.2(\mathrm{CH}), 116$ (arom. $\left.\mathrm{CH}\right), 124$ (arom. CH), 131 (arom. CH), 133.1 (arom. CH), 134.5 (quat. C), 138.6(quat. C), 147. (quat. C), 142.6 (quat. C), 165.5; (carbonyl C); ${ }^{13} \mathrm{C}-\mathrm{NMR}(400 \mathrm{MHz}, \mathrm{CDCl} 3$, TMS): $\delta 49.05\left(\mathrm{CH}_{2}\right), 53.2\left(\mathrm{CH}_{2}\right), 63.1\left(\mathrm{CH}_{2}\right)$, 115(arom. CH), 125.8 (arom. CH), 130.4 (arom. CH), 128.3 (quat. C), 135.9 (quat. C), 141.5 (quat. C), 147.3 (quat. C), 175.3 (carbonyl C) ; ESI-MS m/ z:424,395,319,235,181.Anal. Calcd. For $\mathrm{C}_{27} \mathrm{H}_{28} \mathrm{~N}_{4} \mathrm{O}$ (424): C, 76.39; H, 6.65; N, 13.20; O, 3.77 Found: C, 76.39; H, 6.65; N, 13.20; O, 3.77.

(4-((5-chloro-1H-benzo[d]imidazol-2-yl) methyl) piperazin-1-yl)(Phenyl) methanone (11j)

Compound $11 \mathrm{j}$ was obtained as a white solid (yield: 52\%; MP:293-295; IR (KBr): $3224(\mathrm{~N}-\mathrm{H})$, $2361(\mathrm{C}-\mathrm{H}), 1672(\mathrm{C}=\mathrm{O}), 1607(\mathrm{C}=\mathrm{N}), 1553(\mathrm{C}=\mathrm{C})$, $1235(\mathrm{C}-\mathrm{N}) \mathrm{cm}^{-1}$; ${ }^{1} \mathrm{H}$ NMR (300 MHz, $\mathrm{CDCl}_{3}$ ) $\delta$ ppm: 3.39-3.41(m, 4H,PIP-H), 4.28(s, $\left.2 \mathrm{H},-\mathrm{CH}_{2}-\right)$, 4.82(s, $1 \mathrm{H}, \mathrm{N}-\mathrm{H}), 7.25-7.85$ (m, 3H, Ar-H of Benzim) 7.98$8.12\left(4 \mathrm{H}, \mathrm{Ar}-\mathrm{H}\right.$ of benzoyl) ; ${ }^{13} \mathrm{C}-\mathrm{NMR}(400 \mathrm{MHz}$, $\left.\mathrm{CDCl}_{3}, \mathrm{TMS}\right): \delta 54.8\left(\mathrm{CH}_{2}\right), 56\left(\mathrm{CH}_{2}\right), 65.2\left(\mathrm{CH}_{2}\right)$, 119(arom. CH), 121.3 (arom. CH), 127.5 (arom. $\mathrm{CH}$ ), 138.5 (quat. C), 140.8 (quat. C), 147.1 (quat. C), 172.4 (carbonyl C).ESI-MS m/z : 354,319,203,151.Anal. Calcd. For $\mathrm{C}_{19} \mathrm{H}_{19} \mathrm{CIN}_{4} \mathrm{O}$ (354): C, 64.31; H, 5.40; Cl, 9.99; N, 15.79; O, 4.51Found: C, 64.28; H, 5.42; Cl, $9.95 ; \mathrm{N}, 15.74 ; \mathrm{O}, 4.48$.

(4-(1-) 5-chloro-1H-benzo[d]imidazol-2yl)ethyl)piperazin-1-yl)(Phenyl) methanone (11k) Compound (11k) was obtained as a white solid (yield: 56\%; MP:245-246; IR (KBr): 3209 (N$\mathrm{H}), 2358(\mathrm{C}-\mathrm{H}), \quad 1667(\mathrm{C}=\mathrm{O}), 1611(\mathrm{C}=\mathrm{N})$,
1557(C=C), 1235 (C-N) cm -1; ${ }^{1} \mathrm{H}$ NMR (300 MHz, CDCl3) $\delta$ ppm:1.22(d,1H,-CH-), 3.24-3.29(m, 4H,PIP-H), 4.09(q, 3H,-CH3), 5.14(s, 1H, N-H), 7.25$7.48(\mathrm{~m}, 4 \mathrm{H}, \mathrm{Ar}-\mathrm{H}$ of Benzim) 7.89-8.20 (4H, Ar - $\mathrm{H}$ of benzoyl) ; ${ }^{13} \mathrm{C}-\mathrm{NMR}\left(400 \mathrm{MHz}, \mathrm{CDCl}_{3}, \mathrm{TMS}\right): \delta$ $18.9\left(\mathrm{CH}_{3}\right), 50\left(\mathrm{CH}_{2}\right), 52.4\left(\mathrm{CH}_{2}\right), 63.4(\mathrm{CH})$, 118(arom. CH), 121.6 (arom. CH), 127.5 (arom. CH), 133.5 (quat. C), 136.2 (quat. C), 143.2 (quat. C), 174.5 (carbonyl C); ESI-MS m/z:368,319,257, 145,117. Anal. Calcd. For $\mathrm{C}_{20} \mathrm{H}_{22} \mathrm{~N}_{4} \mathrm{O}$ (368): C, 65.12; $\mathrm{H}, 5.74 ; \mathrm{Cl}, 9.61$; N, 15.19; O, 4.34.Found: C, 65.09; $\mathrm{H}, 5.72 ; \mathrm{Cl}, 9.59 ; \mathrm{N}, 15.17 ; \mathrm{O}, 4.30$.

(4-((5-chloro-1H-benzo[d]imidazol-2-yl) (phenyl) methyl) piperazin-1-yl)(Phenyl) methanone (11l) Compound (11) was obtained as a white solid (yield: 54\%; MP:214-216; IR (KBr): $3214(\mathrm{~N}-$ $\mathrm{H}), 2352(\mathrm{C}-\mathrm{H}), \quad 1668(\mathrm{C}=\mathrm{O}), 1613(\mathrm{C}=\mathrm{N})$, 1539(C=C), 1236 (C-N) cm-1; ${ }^{1} \mathrm{H}$ NMR (300 MHz, $\left.\mathrm{CDCl}_{3}\right) \delta$ ppm:3.19-3.28(m, 4H,PIP-H), 4.78(s, $1 \mathrm{H}, \mathrm{N}-\mathrm{H}$ ), 5.94(s, 1H, C-H), 7.25-8.12 ( Complex m, $4 \mathrm{H}, \mathrm{Ar}-\mathrm{H}) ;{ }^{13} \mathrm{C}-\mathrm{NMR}\left(400 \mathrm{MHz}, \mathrm{CDCl}_{3}, \mathrm{TMS}\right): \delta 48.05$ $\left(\mathrm{CH}_{2}\right), 51.4\left(\mathrm{CH}_{2}\right), 74(\mathrm{CH}), 116$ (arom. $\left.\mathrm{CH}\right), 127$ (arom. CH),131.5 (arom. CH), 134.3 (arom. CH), 134.8 (quat. C), 139 (quat. C), 145.5 (quat. C), 147.2 (quat. C),170(carbonyl C); ESI-MS m/z:430,395,325, 245. Anal. Calcd. For $\mathrm{C}_{25} \mathrm{H}_{23} \mathrm{CIN}_{4} \mathrm{O}$ (430): C, 69.68; $\mathrm{H}, 5.38$; Cl, 8.23; N, 13.00; O, 3.71Found: C, 69.71; $\mathrm{H}, 5.40 ; \mathrm{Cl}, 8.21 ; \mathrm{N}, 13.11 ; \mathrm{O}, 3.69$.

\section{Invitro Antifungal Activity}

The stock solution of $(1 \mathrm{~m} \mathrm{~mole} / \mathrm{ml})$ compounds (equimolar mixture) was prepared in DMSO and water. To each tube containing sterilized sabouraud's liquid medium $(2 \mathrm{ml}), 2 \mathrm{ml}$ of drug solution were added. Each tube was inoculated with the microorganism and was kept at $30^{\circ} \mathrm{C}$ for 14 days.

The solutions were first tested at the concentration of $0.5 \mathrm{mmole} / \mathrm{ml}$. The sets, which are found active at this concentration, were again tested at concentration of $0.25 \mathrm{mmole} / \mathrm{ml}$. The groups, which were found active, were subjected to serial dilutions. The serial dilutions were made to obtain concentrations $0.5,0.25,0.125,0.0625$, $0.0314,0.0152$ and $0.0076 \mathrm{~m} \mathrm{~mole} / \mathrm{ml}$. Positive control tubes (organism + broth + DMSO) and negative control tubes (broth + drug) were also prepared. Each tube was inoculated with the microorganism. 
All the tubes were incubated at $30^{\circ} \mathrm{C}$ for 14 days. The readings were taken as expressed as $(-)$ if inhibition of growth is seen and (+) if inhibition of growth is not seen. [ Table 1]

\section{CONCLUSION}

In conclusion, we have synthesized series of benzimidazole derivatives and confirmed by ${ }^{1} \mathrm{H}$ NMR, ${ }^{13} \mathrm{C}$ NMR, Infra Red (IR),Mass Spectroscopy (MS), and by elemental analysis. These novel compounds were evaluated for in vitro antifungal activity against $C$. albicans strains using turbidimetric method. Benzimidazole derivative with $\mathrm{N}-1$ methyl, C2 as asymmetric center and C5 chlorine substitutions showed good antifungal activity. The structural difference of these compounds from the Ketoconazole can make them less prone to the development of resistance by pathogenic fungal strain against them. The present investigation opens a new lead for antifungal agents and there is a wide scope for future investigation.

\section{ACKNOWLEDGEMENTS}

The authors are very thankful to the management of MET's Institute of Pharmacy for providing infrastructural facilities to carry out the research work. We are very grateful to BCUD, University of Pune for providing financial assistance to the research work. Also we are very thankful to SAIF, Punjab University for providing analytical instrumentation Facility.

\section{REFERENCES}

1. Fridkin, S.K.; Jarvis, W .R.; Epidemiology of nosocomial fungal infections. Clin Microbiol Rev. 1996,9,499-511.

2. Gallis, H. A.; Drew, R. H.; Pickard, W.W.; Amphotericin B: 30 years of clinical experience.Rev Infect Dis.1990,12,308329.doi: 10.1093/clinids/12.2.308

3. Daniel, J.S.;Christopher, A. H.; Carol, M.S.; Current and Emerging Azole Antifungal Agents.Clin Microbiol Rev.1999; 12,40-79.

4. Moudgal, V.; Little, T.; Boikov, D .; Vazquez, A.J.; Multiechinocandin and MultiazoleResistant Candida parapsilosis Isolates Serially obtained during Therapy for Prosthetic Valve Endocarditis,Antimicrob Agents Chemother. 2005, 49,767-769.

5. Sanguinetti, M.; Sanfilippo, S.; Castagnolo, D.; Sanglard, D.; Posteraro, B.; Donzellini, G.; Botta, M.; Novel Macrocyclic Amidinoureas: Potent Non-Azole Antifungals Active against Wild- Type and Resistant Candida Species. ACS Med Chem Lett. 2013, 9,852-857.

6. Hakan, G.; Canan, K.; Ufuk, A.; Synthesis of 1,2,5(6)-Trisubstituted Benzimidazoles and Evaluation of Their Antimicrobial Activities. 1995,Archiv der Pharmazie,328,425-430.

7. Garuti, L.; Roberti, M.; Gentilomi, G.; Synthesis and antiviral assays of some 2-substituted benzimidazole- $N$-carbamates. II Farmaco 2000, 55, 3539.

8. Kakuta, H.; Sudoh, K.; Sasamata, M.; Yamagishi, S.;Telmisartan has the strongest binding affinity to angiotensin II type 1 receptor: comparison with other angiotensin II type 1 receptor blockers.International Journal of Clinical Pharmacology Research 2005, 25,41-46.

9. Janssens, F.; Torremans, J.; Janssen, M.; Stokbroekx R. A.; Luyckx ,M.;Janssen, P.A.; Synthesis and antihistaminic activity of $\mathrm{N}-(4-$ piperidinyl)-1H-benzimidazol-2-amines. J.Med. Chem. 1985, 28, 1925-1933.

10. Sondhi, S.M.; Singh, N.; Kumar, N.; Lozach, O.; Meijer, L.; Synthesis, anti-inflammatory, analgesic and kinase (CDK-1, CDK-5 and GSK-3) inhibition activity evaluation benzimidazole/benzoxazole derivatives and some Schiff's bases.Bioorganic \& Medicinal Chemistry, 2006,14,3758-3765.

11. Küçükbay, H.; Durmaz, R.; Güven, M.; Günal, S.;Synthesis of some benzimidazole derivatives and their antibacterial and antifungal activities. Arzneimittelforschung; 2001,51,420-4.

12. Canan ,K.; ALTANLAR, N.;Synthesis of Some New Benzimidazole Carbamate Derivatives for Evaluation of Antifungal Activity.Turk $J$ 
Chem. 2003,27, 35 - 39.

13. Garuti, L.; Roberti, M.; Pession, A.; Leoncini, E.; Hrelia, S.; Synthesis and antiproliferative activity of some thiazolylbenzimidazole-4,7diones,Bioorg. Med. Chem. Lett. 2001,11,3147-3149.

14. Li GR, Liu J.; Pan Q.; Song Z. B.; Luo F.L.; Wang, S.R.; Zhang X.L.; Zhou, X.; Synthesis and anti-HIV activity of [ddN]-[ddN] dimers and benzimidazole nucleoside dimers.Chem Biodivers. 2009 , 6, 2200-8.

15. Edward, R. C.; George, C.; Abdus, S. S.; Antioxidant properties of benzimidazoles $\mathrm{J}$. Agric. Food Chem. 1974, 22 , 918-918.

16. Ravishankara, D.K.; Chandrashekara, P.G.; Synthesis of some novel benzimidazole derivatives and it's biological evaluation. Eur J.Chem. 2012, 3, 359-362.

17. Zhang, H.; Guri , V. D.; Gui-Xin, C.; ChengHe Z.; Design, synthesis and antimicrobial evaluation of novel benzimidazole type of Fluconazole analogues and their synergistic effects with Chloromycin, Norfloxacin and Fluconazole,Eur. J. Med. Chem. 2012, 55, 164-175.

18. Chaudhary, P.; Kumar, R.; Akhilesh K. V.; Devender S., Yadav V.; Chhillar, A.K.; Sharmab G.L.; Chandra, R.; Piperazine: Synthesis and molecular modeling, Bioorganic \& Medicinal Chemistry, 2006,12, 1819-1826
19. Sivakumar, R.; Basha S. N.; Kumarnallasivan P.; Vijaianand P. R.; Pradeepchandran, R.; Jayaveera K. N.; Venkatnarayanan R. A.; Computational design and docking studies on Escherichia coli b-Ketoacyl-Acyl carrier protein synthese III using auto dock. J. Pharm. Res.,2010, 3,1460-1462.

20. Surendrareddy, K.; Dushyanth, S.; Elango, $\mathrm{K}$.; Microwave assisted synthesis of some novel benzimidazole substituted Fluoroquinolones and their antimicrobial evaluation, J. Pharm. Sci. Res. 2010,2, 6976.

21. Mavrova, A.T., Vuchev, D., Anichina, K., Vassilev, N., Synthesis, antitrichinnellosis and antiprotozoal activity of some novel thieno[2,3-d]pyrimidin-4(3H)-ones containing benzimidazole ring. Eur. J. Med. Chem. 2010. 45, 5856.

22. Willitzer, H; Bräuniger, H; Engelmann, D; Krebs D,Ozegowski, W; Tonew, M.;Synthesis and antiviral effect of substituted 5-ureido and 5-thioureidobenzimidazole derivatives. Pharmazie. 1978,33,30-38.

23. Rammohan, P.; Recent progress of Ammonium chloride as catalyst in organic synthesis Journal of Applied Chemistry, 2013, 4, 86-99.

24. Cappucino, J.G.; and Sherman, N.; Microbiology: A laboratory manual, $4^{\text {th }}$ edition, Addison-Wesley longman, Inc., New York, 1996,199. 